\title{
DETERMINING $\Omega_{0}$ AND $\lambda_{0}$ FROM COSMOLOGICAL REDSHIFT DISTORTION OF GALAXIES AND QUASARS
}

\author{
YASUSHI SUTO \\ Department of Physics, \\ and Research Center for the Early Universe (RESCEU) \\ The University of Tokyo, Tokyo 113, Japan
}

\section{Introduction}

The three-dimensional distribution of galaxies in the redshift surveys differ from the true one since the distance to each galaxy cannot be determined by its redshift $z$ only; for $z \ll 1$ the peculiar velocity of galaxies, typically $(100-1000) \mathrm{km} / \mathrm{sec}$, contaminates the true recession velocity of the Hubble flow, while the true distance for objects at $z \gtrsim 1$ sensitively depends on the (unknown and thus assumed) cosmological parameters. This hampers the effort to understand the true distribution of large-scale structure of the universe. Nevertheless such redshift-space distortion effects are quite useful since through the detailed theoretical modeling, one can derive the peculiar velocity dispersions of galaxies as a function of separation, and also can infer the cosmological density parameter $\Omega_{0}$, the dimensionless cosmological constant $\lambda_{0}$, and the spatial biasing factor $b$ of galaxies and/or quasars, for instance. In this talk, I discuss the importance of such redshift distortion induced by the geometry of the universe, which summarizes the recent results of my collaborative work in this topic (Matsubara \& Suto 1996; Nakamura, Matsubara, \& Suto 1998; Magira, Matsubara, Jing, \& Suto 1998).

\section{Cosmological redshift distortion}

Let us consider a pair of objects located at redshifts $z_{1}$ and $z_{2}$ whose redshift difference $\delta z \equiv z_{1}-z_{2}$ is much less than the mean redshift $z \equiv\left(z_{1}+z_{2}\right) / 2$. Then the observable separations of the pair parallel and perpendicular to the line-of-sight direction, $s_{\|}$and $s_{\perp}$, are given as $\delta z / H_{0}$ and $z \delta \theta / H_{0}$, re- 


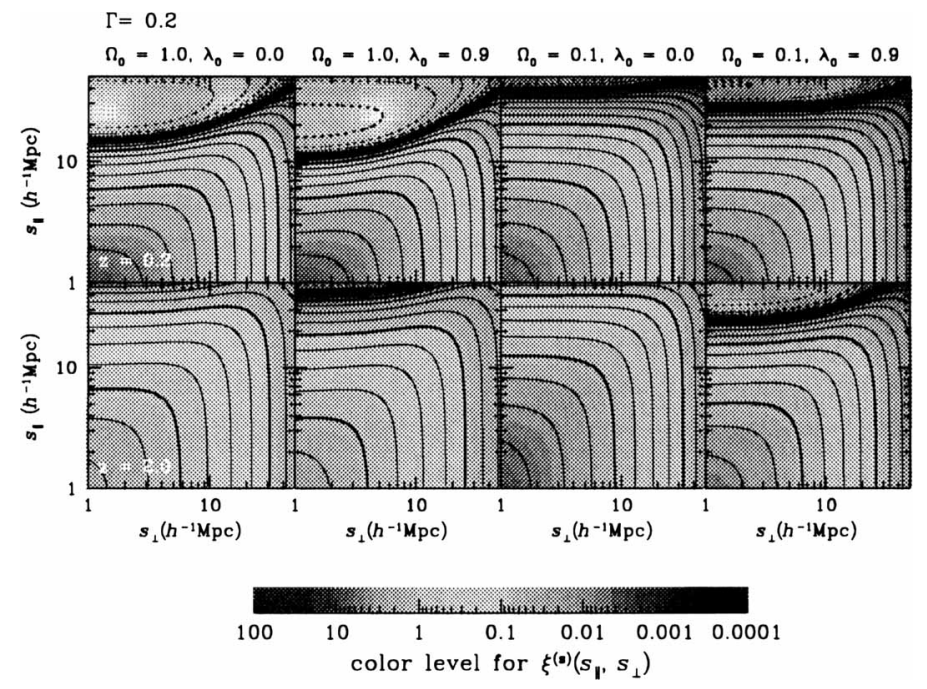

Figure 1. Solid and dashed lines indicate the positive and negative $\xi^{(s)}$, respectively. Contour spacings are $\Delta \log _{10}|\xi|=0.25$. Upper: $z=0.2$, Lower: $z=2$.

spectively, where $H_{0}$ is the Hubble constant and $\delta \theta$ denotes the angular separation of the pair on the sky. The cosmological redshift-space distortion originates from the anisotropic mapping between the redshift-space coordinates, $\left(s_{\|}, s_{\perp}\right)$, and the real comoving ones $\left(x_{\|}, x_{\perp}\right) \equiv\left(c_{\|} s_{\|}, c_{\perp} s_{\perp}\right)$; $c_{\perp}$ is written as $c_{\perp}=H_{0}(1+z) D_{A} / z$ in terms of the angular diameter distance $D_{A}$, and

$$
c_{\|}(z)=\frac{H_{0}}{H(z)}=\frac{1}{\sqrt{\Omega_{0}(1+z)^{3}+\left(1-\Omega_{0}-\lambda_{0}\right)(1+z)^{2}+\lambda_{0}}}
$$

(Alcock \& Paczyński 1979; Matsubara \& Suto 1996).

The relation between the two-point correlation functions of quasars in redshift space, $\xi^{(s)}\left(s_{\perp}, s_{\|}\right)$, and that of mass in real space, $\xi^{(r)}(x)$, can be derived in linear theory:

$$
\begin{aligned}
& \xi^{(s)}\left(s_{\perp}, s_{\|}\right)=\left(1+\frac{2}{3} \beta(z)+\frac{1}{5}[\beta(z)]^{2}\right) \xi_{0}(x) P_{0}(\mu) \\
& -\left(\frac{4}{3} \beta(z)+\frac{4}{7}[\beta(z)]^{2}\right) \xi_{2}(x) P_{2}(\mu)+\frac{8}{35}[\beta(z)]^{2} \xi_{4}(x) P_{4}(\mu),
\end{aligned}
$$

where $x \equiv \sqrt{c_{\|}^{2} s_{\|}^{2}+c_{\perp}^{2} s_{\perp}^{2}}, \mu \equiv c_{\|} s_{\|} / x, P_{n}$ 's are the Legendre polynomi- 


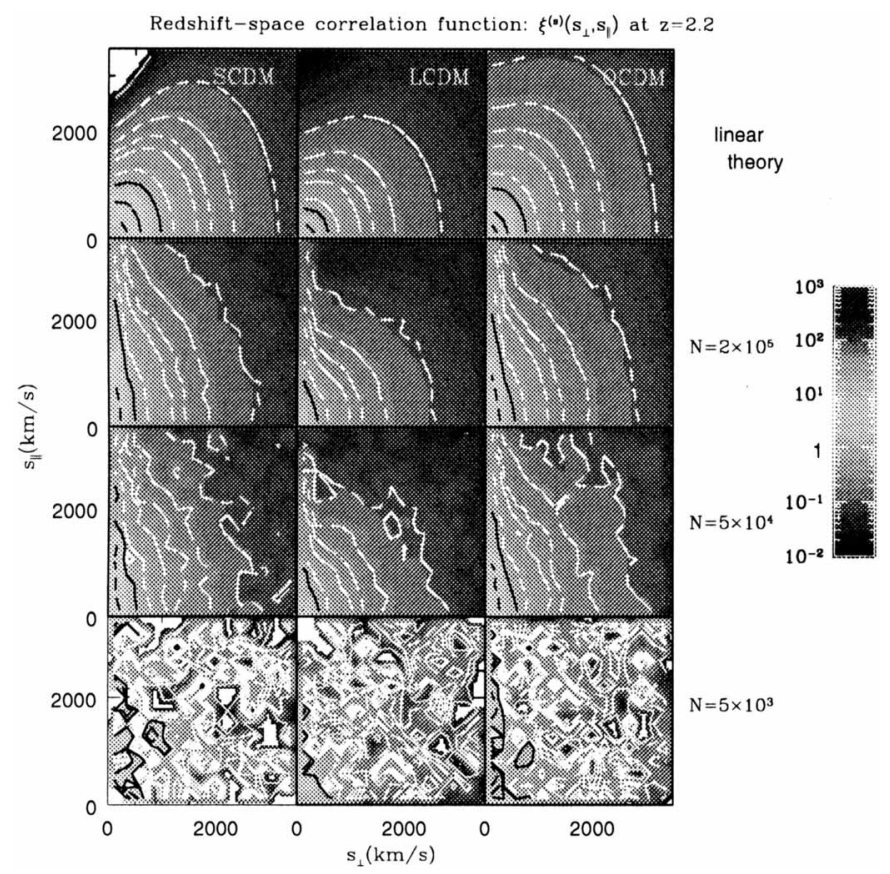

Figure 2. $\xi^{(s)}\left(s_{\perp}, s_{\|}\right)$from linear theory (Upper) and N-body simulations (Lower) at $z=2.2$.

als,

$\beta(z) \equiv \frac{1}{b(z)} \frac{d \ln D(z)}{d \ln a}, \quad \xi_{2 l}(x)=\frac{(-1)^{l}}{x^{2 l+1}}\left(\int_{0}^{x} x d x\right)^{l} x^{2 l}\left(\frac{d}{d x} \frac{1}{x}\right)^{l} x \xi^{(r)}(x)$,

and $D(z)$ is the linear growth rate. Figure 1 shows the above prediction in linear theory for four models with the fixed shape parameter $\Gamma=0.2$ and the fluctuation amplitude $\sigma_{8}=1$ assuming no bias, i.e., $b(z)=1$. In fact, the above expression by Matsubara \& Suto (1996) is the generalization of the $z=0$ result of Hamilton (1992; see also Kaiser 1987) at an arbitrary $z$. The essentially identical formula, but for $P(k)$, was independently derived and discussed by Ballinger, Peacock \& Heavens (1996).

In reality, however, the observable two-point correlation functions would be also contaminated by non-linear peculiar velocity as well as statistically limited by the available number of tracer objects. To incorporate the nonlinear effect, we compute $\xi^{(s)}\left(s_{\perp}, s_{\|}\right)$from a series of N-body simulations in CDM models with $N=256^{3}$ particles in $\left(300 h^{-1} \mathrm{Mpc}\right)^{3}$ box (Magira et al. 1998). Figure 2 shows several examples at $z=2.2$ for SCDM, OCDM, and LCDM models which have $\left(\Omega_{0}, \lambda_{0}, \Gamma, \sigma_{8}\right)=(1,0,0.5,1.2),(0.3,0,0.25,1.0)$, and $(0.3,0.7,0.21,1.0)$, respectively. The degree to the extent which one can 


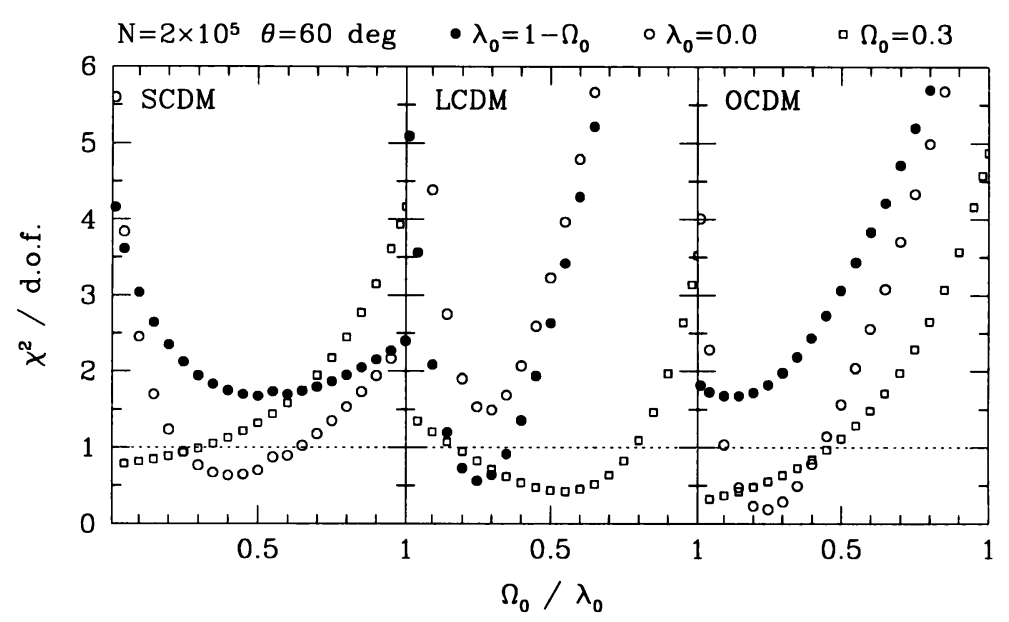

Figure 3. $\chi^{2}$ analysis from the $\xi\left(s_{\perp}, s_{\|}\right)$curve fit using $N=2 \times 10^{5}$.

recover the underlying correlation of small amplitudes is sensitive to the sampling rate, or the number density of objects. In computing $\xi^{(s)}\left(s_{\perp}, s_{\|}\right)$ from the simulation data, we reduce the number density of objects systematically by randomly sampling simulation particles. Figure 3 plots the reduced $\chi^{2}$ for the fit of $\xi^{(s)}\left(s_{\perp}, s_{\|}\right)$from simulations to the linear theory prediction. In performing the fit, we exclude the regions with $s_{\|} / s_{\perp}>2$ which are supposed to be seriously contaminated by nonlinear peculiar velocities.

While Figures 3 demonstrates that the current methodology works in principle, Figure 2 clearly shows that the expected $\xi^{(s)}\left(s_{\perp}, s_{\|}\right)$for unbiased tracers is quite noisy for realistic number density of quasars at the corresponding redshift; the number density of objects $\gtrsim 0.004 h^{3} \mathrm{Mpc}^{-3}$ is required to discriminate models with $\Omega_{0}=1$ and $\Omega_{0}=0.3$ on the basis of this straightforward comparison, which is roughly more than one order of magnitude larger than the observed quasars (Boyle, Shanks, \& Peterson 1988). This situation would be significantly improved since quasars are (and high- $z$ galaxies) are likely to be biased tracers; Franca, Andreani, \& Cristiani (1998), for instance, reported a correlation length $6.2 \pm 1.6 h^{-1} \mathrm{Mpc}$ (comoving) from 388 quasars at an average redshift 1.3. Such a high degree of clustering significantly increases the $\mathrm{S} / \mathrm{N}$ of $\xi^{(s)}$ and results in stronger constraints from the current test (c.f., Magira et al. 1998).

\section{Implication for galaxy redshift survey}

The cosmological distortion effect becomes important also even for shallower redshift surveys like SDSS (Gunn \& Weinberg 1995) and 2dF. One 


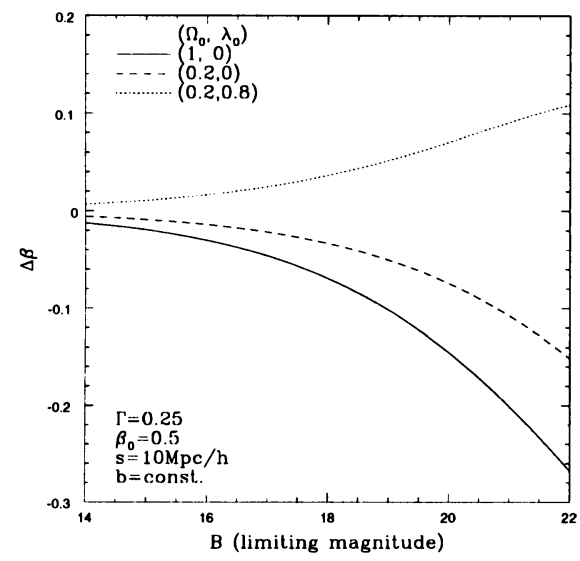

Figure 4. Systematic deviation $\Delta \beta$ in the estimates of $\beta_{0}=\Omega_{0}^{0.6} / b$ versus the limiting magnitude of redshift surveys in $\mathrm{B}$ band $\left(\beta_{0}=0.5\right)$.

may formally expand $\xi^{(s)}\left(s_{\perp}, s_{\|}\right)$in terms of the observables, $s \equiv \sqrt{s_{\|}^{2}+s_{\perp}^{2}}$ and $\mu_{s} \equiv s_{\|} / s$, instead of the unobservable variables $\left(x(z), \mu_{x}(z)\right)$ :

$$
\xi^{(s)}\left(s_{\perp}, s_{\|} ; z\right)=\sum_{l=0}^{2} \xi_{2 l}(x ; z) P_{2 l}\left(\mu_{x}\right)=\sum_{l=0}^{\infty} \zeta_{2 l}(s ; z) P_{2 l}\left(\mu_{s}\right) .
$$

Since we are interested in $z \ll 1$ galaxy redshift surveys, we can further expand the above summation up to the first order in $z$, and then obtain

$$
\xi^{(s)}\left(s_{\perp}, s_{\|} ; z\right) \approx \sum_{l=0}^{3} \zeta_{2 l}(s ; z) P_{2 l}\left(\mu_{s}\right) .
$$

This explicit expression enables us to estimate a systematic bias for $\beta_{0}$ on the basis of Hamilton's method (Hamilton 1992). The result (Nakamura et al. 1998) consists of the two terms corresponding to the evolution of $\beta$-parameter and the geometrical effect:

$$
\begin{gathered}
\left.\frac{d \beta_{\text {est }}}{d z}\right|_{z=0} ^{\text {sys }}=\left.\frac{d \beta(z)}{d z}\right|_{z=0}+\frac{1+q_{0}}{1+\frac{6 \beta_{0}}{7}+\frac{3 \beta_{0}^{2}}{35}} \times\left[\frac{\beta_{0}}{7}\left(1-\frac{\beta_{0}}{5}-\frac{11 \beta_{0}^{2}}{15}-\frac{\beta_{0}^{3}}{7}\right)\right. \\
\left.+\frac{1}{4}\left(1+\frac{12 \beta_{0}}{7}+\frac{34 \beta_{0}^{2}}{35}+\frac{4 \beta_{0}^{3}}{21}+\frac{\beta_{0}^{4}}{49}\right) \frac{\partial \xi / \partial \ln s}{\bar{\xi}-\xi}\right]
\end{gathered}
$$

where $q_{0}$ is the deceleration parameter $\left(=\Omega_{0} / 2-\lambda_{0}\right)$. Figure 4 displays the systematic bias for $\beta_{0}$ in $\Gamma=0.25$ models with different $\Omega_{0}$ and $\lambda_{0}$. The 
resulting systematic bias is smaller than the statistical errors of the existing surveys (e.g., Ratcliffe et al. 1996), but is expected to be larger than those of the next-generation, deep and wide redshift surveys including SDSS and $2 \mathrm{dF}$.

\section{Conclusions}

Observable spatial distribution of galaxies and quasars is necessarily distorted by the peculiar velocity of objects and by the geometry of the universe itself. In this talk, I have discussed this cosmological redshift-space distortion, especially keeping in mind the application to the future surveys of galaxies and quasars. This effect can be regarded as either noises or signals depending on what you are interested in; if you are interested in determining the real-space correlation functions of high-z objects or the $\beta_{\text {gal }}(z=0)$, the effect is a noise which should be corrected for. If you would like to determine the cosmological parameters such as $\Omega_{0}, \lambda_{0}$, and $b_{\mathrm{QSO}}(z)$, however, the cosmological redshift-space distortion would provide important signals in a complementary fashion to the constraints from the cosmic microwave background anisotropies.

The present talk is based on my collaborative work with Y.P. Jing, H. Magira, T. Matsubara, and T.T.Nakamura. Numerical computations were carried out on VPP300/16R and VX/4R at the Astronomical Data Analysis Center of the National Astronomical Observatory, Japan, as well as at RESCEU (Research Center for the Early Universe, University of Tokyo) and KEK (National Laboratory for High Energy Physics, Japan). This research was supported in part by the Grants-in-Aid by the Ministry of Education, Science, Sports and Culture of Japan (07CE2002) to RESCEU, and by the Supercomputer Project (No.97-22) of High Energy Accelerator Research Organization (KEK).

\section{References}

Alcock, C., \& Paczyński, B. 1979, Nature, 281, 358

Ballinger, W.E., Peacock, J.A., \& Heavens, A.F. 1996, MNRAS, 282, 877

Boyle, B.J., Shanks, T. \& Peterson, B.A. 1988, MNRAS, 235, 935

Franca, F.L., Andreani, P., \& Cristiani, S. 1998, ApJ, in press (astro-ph/9711048)

Gunn, J.E., \& Weinberg, D.H. 1995, in Wide Field Spectroscopy and the Distant Universe, eds. S.J. Maddox \& A. Araǵon-Salamanca (World Scientific, Singapore), p.3

Hamilton, A.J.S. 1992, ApJ, 385, L5

Kaiser, N. 1987, MNRAS, 227, 1

Magira, H., Matsubara, T., Jing, Y.P. \& Suto, Y. 1998, in preparation

Matsubara, T., \& Suto, Y. 1996, 470, L1

Nakamura, T.T., Matsubara, T., \& Suto, Y. 1998, 493, in press

Ratcliffe, A., et al. 1996, MNRAS, 281, L47 